\title{
Économie et institutions
}

E Institutions

26 | 2017

Approches institutionnalistes de la monnaie

Une conception matérialiste des prix ne suppose nullement d'adhérer à la théorie de la valeur travail

Nouvelle Réponse à Jean-Marie Harribey

André Orléan

CpenEdition

Journals

Édition électronique

URL : http://journals.openedition.org/ei/6092

DOI : 10.4000/ei.6092

ISSN : 2553-1891

Éditeur

Association Économie et Institutions

Référence électronique

André Orléan, « Une conception matérialiste des prix ne suppose nullement d'adhérer à la théorie de la valeur travail », Économie et institutions [En ligne], 26 | 2017, mis en ligne le 03 juillet 2018, consulté le 02 mai 2019. URL : http://journals.openedition.org/ei/6092 ; DOI : 10.4000/ei.6092

Ce document a été généré automatiquement le 2 mai 2019.

Revue Économie et institutions 


\section{Une conception matérialiste des prix ne suppose nullement d'adhérer à la théorie de la valeur travail}

Nouvelle Réponse à Jean-Marie Harribey

\section{André Orléan}

Dans son « Examen critique de la vision autoréférentielle de la valeur et de la monnaie », Jean-Marie Harribey accorde une place importante à mes travaux personnels comme à ceux que j'ai développés avec Michel Aglietta ${ }^{1}$. Si les critiques qu'il m'adresse sont nombreuses et fortes, elles sont formulées cependant avec retenue, sans volonté de nuire mais, tout au contraire, avec le souci de faire émerger de possibles terrains d'entente. Cela est particulièrement visible, me semble-t-il, pour ce qui est de l'approche institutionnaliste de la monnaie qu'il ne rejette pas. Cette mesure dans la critique est une qualité tout à fait appréciable en ce qu'elle incite celui qui est critiqué à poursuivre plus avant la discussion, comme en témoigne la présente contribution. Il ne faut cependant pas perdre de vue le fait que la question de la monnaie ne constitue nullement, pour JeanMarie Harribey, le cœur de la controverse. L'essentiel de ce qui motive son profond et tenace $^{2}$ désaccord à mon endroit est ailleurs : dans mon rejet déclaré ${ }^{3}$ de la théorie de la valeur marxiste. C'est bien cette question qu'on trouve au centre du texte que propose aujourd'hui Jean-Marie Harribey. Il s'agit, pour lui, de montrer que l'abandon de la théorie de la valeur travail est intenable parce qu'il conduirait à couper la détermination des prix - prix des marchandises comme prix des actifs financiers - des conditions sociales et techniques de la production. Par conséquent, dès lors que Jean-Marie Harribey est prêt à accepter l'approche institutionnaliste de la monnaie, la question qu'il se pose est de savoir si cette acceptation conduit nécessairement à l'abandon de la valeur travail, ce à quoi il se refuse catégoriquement : « Notre objectif est d'examiner si une conception renouvelée de la monnaie autour de l'idée qu'elle est une institution sociale "instituante", 
et non pas qui résulte des échanges, implique obligatoirement le rejet de toute théorie de la valeur. "

Dans cette brève contribution, nous chercherons à montrer que la thèse qui est au cœur de la critique que m'adresse Jean-Marie Harribey, thèse selon laquelle l'abandon de la théorie marxiste de la valeur imposerait de rompre avec ce que je propose de nommer une "conception matérialiste des prix» - à savoir une approche qui se refuse à déconnecter les prix des marchandises comme des actifs financiers des conditions de la production - est entièrement fausse. Pour que ne subsiste aucune ambiguïté à ce sujet, indiquons d'emblée, de la manière la plus formelle, que je partage totalement la position de Jean-Marie Harribey selon laquelle le prix des marchandises, comme celui des actifs financiers, ne saurait être indépendant des conditions sociales et techniques de production. Je vois d'ailleurs mal comment une position contraire serait possible! Pourtant c'est bien la position que Jean-Marie Harribey m'attribue. Il me reproche, par conséquent, d'être incapable d'expliquer "qu'un Airbus vaille 500 millions d'euros, un sous-marin 2 milliards d'euros et une douzaine d'œufs 4 euros ». La présente contribution a pour but principal de dissiper ce malentendu, je l'espère une bonne fois pour toutes. J'essaierai de montrer qu'une théorie matérialiste des prix ne nécessite nullement d'adhérer à la loi de la valeur telle qu'elle est communément interprétée par la tradition marxiste ${ }^{4}$.

Je commencerai mon argumentation en m'intéressant à la question de la formation des cours boursiers avant d'en venir à la question du prix des marchandises. Cependant, avant de présenter ces arguments, il me faut reconnaître ma part de responsabilité, ayant certainement manqué de clarté dans l'explicitation de ma position, comme en atteste le fait que Jean-Marie Harribey n'a pas été le seul à me faire un tel reproche. Je pense en particulier à Laurent Cordonnier (2012) - que cite Jean-Marie Harribey - qui s'avoue également frustré par le fait que, dans L'Empire de la valeur, je ne dis rien sur la détermination des prix. Cela le conduit à une question proche de celle que me pose JeanMarie Harribey: «Pourquoi les tomates en grappe valent-elles 4,50 euros le kilo [...] et pourquoi un sac à main de luxe vaut-il 600 euros ? $\|^{5} \mathrm{Je}$ pense également à Matthieu Montalban (2012) qui me fait ce même reproche de ne pas proposer une théorie des prix. Il écrit: "Si l'hétérodoxie se retrouve sans théorie de la valeur, ou simplement sans théorie des prix alternative, on peut naturellement s'interroger quant à sa capacité à produire une représentation de l'économie politique alternative à celle de l'orthodoxie. " Plus loin, après avoir rappelé le rôle central que jouent les prix dans l'allocation marchande des ressources, il observe que: «Sur ces questions, force est de remarquer que l'approche monétaire [d'Orléan] ne nous dit rien, et qu'elle ne peut d'ailleurs rien en dire car tel n'est pas son but. » À l'évidence, il y a là un manque. J'espère que la présente contribution permettra de la combler.

\section{Le rôle des profits dans la détermination des cours boursiers}

4 Commençons par le "plus simple»: la formation des cours boursiers. Jean-Marie Harribey me reproche une conception du marché boursier qui négligerait totalement le rôle des fondamentaux : « le point ultime du raisonnement sur l'autonomie de la finance est que non seulement les fondamentaux ne déterminent plus les valeurs financières, 
mais que la relation est inversée: ce sont les croyances qui façonneraient les fondamentaux ». On ne saurait être plus clair. Autrement dit, mon approche ignorerait totalement le rôle des fondamentaux et, en particulier, celui des conditions de production. Tout serait affaire de croyances. Qu'en est-il ?

En matière financière, ma thèse centrale, qui distingue radicalement mon approche de celle proposée par la théorie néoclassique, est qu'il n'existe pas quelque chose comme une valeur fondamentale objective dont le cours boursier serait le reflet fidèle ${ }^{6}$ (hypothèse d'efficience financière). Pour autant, cela ne signifie pas que les cours soient déconnectés des profits. Ce qui importe pour l'évaluation boursière d'une entreprise reste toujours, à mes yeux, les profits futurs, mais mon approche intègre le fait qu'en raison de l'incertitude radicale, la profitabilité future n'est pas une grandeur objective qu'on pourrait déduire mécaniquement de telle ou telle observation du monde réel. Il revient au marché d'en proposer une estimation par le biais de ce que j'appelle une convention financière. En effet, selon les périodes, le marché n'analyse pas d'une manière identique la capacité des entreprises à faire des profits. Par exemple, le marché a pu privilégier, à une époque, les conglomérats et, à un autre moment, les entreprises focalisées sur leur cœur de métier. Tel est mon apport: intégrer le fait qu'anticiper les profits suppose un travail conceptuel interprétatif au travers d'hypothèses qui sont puisées dans les croyances de l'époque. En conséquence, il n'est pas rare que le marché se trompe. La convention elle-même peut être mise également en défaut lorsqu'elle est démentie par les faits; ce que nous avons nommé les "anomalies». Par exemple, les difficultés qu'a rencontrées le commerce électronique fin 1999 et début 2000 ont conduit à la remise en cause de la convention financière de l'époque et à l'éclatement de la bulle internet en mars 2000. C'est dire que je ne conçois pas l'autonomie de la valorisation financière comme s'identifiant à une pure autoréférentialité, ce que Jean-Marie Harribey nomme, dans la citation précédente, «le point ultime de l'autonomie». Par conséquent, à la question que pose Jean-Marie Harribey : «La bulle ne s'est-elle pas effondrée parce que justement l'écart [entre la valorisation boursière et les profits réels] était intenable? », je réponds comme lui "Oui», avec seulement cet addendum que l'évaluation des profits à venir pour les firmes Internet reposait sur une convention qui incluait bien d'autres variables que la seule observation des profits passés et présents, y compris des variables dont le lien avec les profits pouvait être des plus ténus, par exemple l'évolution du nombre de visiteurs - ou de clics - pour les diverses start-up, sans quoi d'ailleurs le mouvement haussier qu'ont connu des entreprises structurellement déficitaires serait largement incompréhensible.

6 Rappelons par ailleurs que, pour faire comprendre ce qu'est la convention financière, $L e$ Pouvoir de la finance la compare au paradigme au sens de Kuhn. C'est d'ailleurs une des hypothèses les plus originales et les plus centrales du livre. Or, ce rapprochement montre à lui seul, sans aucune ambiguïté, que ce que je cherche à penser n'est absolument pas "un monde enchanté, fétichisé [...] se valorisant tout seul», mais bien une activité collective d'analyse ayant pour finalité la connaissance du monde économique. Il s'agit de faire valoir que cette connaissance ne se réduit pas à l'observation routinière de quelques grandeurs prédéterminées mais suppose une activité interprétative collective reposant sur la construction d'hypothèses, par ailleurs largement influencées par les croyances de l'époque, quant à la nature du contexte économique. Il est vrai qu'en présentant ce résultat, j'ai d'abord eu le souci de me différencier de l'hypothèse d'efficience financière qui alors - en 1999 - régnait en maître, ce qui m'a conduit à mettre surtout l'accent sur 
l'autonomie de la finance dans la construction des conventions financières. Cependant, il n'est pas possible de soutenir que l'approche conventionnaliste que j'ai proposée ignore - ou même sous-estime - le rôle des fondamentaux dans l'évaluation financière. Il suffit d'ailleurs de lire le passage suivant dans Le Pouvoir de la finance pour s'en convaincre :

Les analyses qui ont été menées jusqu'à maintenant à propos des valeurs Internet et des situations mexicaine et asiatique nous conduisent à formuler deux nouvelles hypothèses quant à la nature de la convention financière : la convention repose sur un modèle d'interprétation de type fondamentaliste ( $j e$ souligne) et sa structure est identique à celle du paradigme chez Thomas Kuhn. Présentons-les successivement.

Les exemples précédents montrent que les évaluations conventionnelles reposent toujours sur un diagnostic fondamentaliste. Qu'il s'agisse d'une entreprise, d'un secteur ou d'un pays, les conventions se focalisent sur la croissance à venir ou sur les profits futurs et justifient de cette façon leurs estimations des prix boursiers. C'est le rôle directeur [des fondamentaux] dans l'évaluation financière qui est ici reconnu: si les investisseurs ne doutent pas qu'une multitude de facteurs les plus divers puisse influencer les prix d'actif, ils n'en sont pas moins convaincus que la valeur boursière doit être un reflet fidèle des données économiques réelles pour être légitime. Cependant, comme on l'a déjà souligné, il est impossible d'estimer correctement une grandeur qui dépend si fortement d'une multitude de facteurs économiques imprévisibles. La convention procède alors par hiérarchisation : elle privilégie certains facteurs et en néglige d'autres [...] Il s'ensuit que l'évolution historique des conventions boursières s'analyse comme une succession de modèles interprétatifs, chaque modèle se définissant par la variable ou le mécanisme sur lequel il met l'accent : pour telle période, ce fut le déficit commercial américain qui a dominé les anticipations; puis, pour telle autre, ce fut sur la croissance de la masse monétaire ${ }^{7}$.

7 «La convention repose sur un modèle d'interprétation de type fondamentaliste » : on ne saurait dire les choses plus clairement. Tel est mon point de vue. Ayant le privilège d'avoir un accès direct à ce que je pense, je peux assurer le lecteur sceptique que telle est bien ma position. Ceci devrait clore ce malentendu : l'autonomie de la finance que je défends n'est en rien une pure indépendance.

8 En appui à ses critiques, Jean-Marie Harribey cite cependant plusieurs passages que j'ai écrits et qui semblent aller dans le sens d'une position toute contraire, le plus saisissant étant certainement celui-ci : «On retrouve ici le caractère clos du monde de la finance qui détermine ce que sont les fondamentaux à partir des interprétations qu'il produit. Les valeurs fondamentales n'existent pas en elles-mêmes. Il n'y a que des interprétations. $»^{8}$ N'est-ce pas très exactement ce que me reproche Jean-Marie Harribey? Commençons par reconnaître que cette formulation est malheureuse. Cependant, pour ma défense, il convient de souligner que cette analyse porte explicitement sur un «ici » qu'il s'agit d'avoir à l'esprit quand on la lit ${ }^{9}$. En effet, ce passage traite d'un phénomène tout à fait particulier, et non pas de la finance en général, à savoir la dynamique spéculative baissière - par exemple, la chute brutale qui s'opère au moment d'un krach - pour laquelle, en effet, il est vrai que la composante autoréférentielle domine l'évaluation des profits. Le fait de croire majoritairement à la baisse produit alors une baisse généralisée, très largement déconnectée des fondamentaux, donnant lieu à un phénomène de surréaction. Les exemples en sont multiples, sur les marchés boursiers comme sur les marchés des changes. On peut citer comme exemple le cas du peso juste après la crise argentine de décembre 2001. Autrement dit, cette analyse ne prétend qu'à une portée étroitement circonscrite, comme l'indique clairement le titre de la section dans laquelle 
elle prend place : «Les dynamiques baissières et la crise $»^{10}$. Ce phénomène a pour origine la rupture de la convention, " phénomène intégralement négatif qui met les investisseurs face à une perte généralisée de leurs repères [...] C'est la défiance pure qui se donne libre cours $»^{11}$. La citation précédemment mise en avant par Jean-Marie Harribey n'est valide que pour ces épisodes extrêmes de rupture des conventions. Et il me semble que, concernant ces mouvements de panique, elle n'est pas sans fondement.

Deux autres citations sont produites par Jean-Marie Harribey à l'appui de sa démonstration mais qui, me semble-t-il, se comprennent aisément sans soulever de questions concernant le rôle des fondamentaux. La première citation - «[...] si l'on doit reconnaître que le capital est la source créatrice de toute valeur, aucun capital en particulier ne possède en lui-même la capacité de l'affirmer. Son aptitude à créer de l'utilité sociale, autrement dit sa valeur sociale, seul le marché en juge $»^{12}$ - est tirée d'un chapitre, intitulé «L'individualisme patrimonial», qui porte spécifiquement sur le capitalisme financier contemporain et ses caractéristiques. Il s'agit d'observer que, dans le cadre de ce nouveau capitalisme, le cours boursier accède à une dignité qu'il ne connaissait pas à l'époque du fordisme, à savoir devenir l'évaluation de référence. Il me semble qu'il en est effectivement ainsi : le marché financier a acquis, dans le capitalisme néolibéral, un rôle dominant comme en atteste le rôle que joue, dans la gestion des entreprises, la valeur actionnariale. Notons que cette proposition n'impose en tant que telle aucune hypothèse quant à l'autonomie de l'évaluation financière. Elle en est largement déconnectée et, en conséquence, n'apporte pas d'élément au débat concernant l'impact des fondamentaux sur la valorisation boursière. La seconde citation - dès lors, la finance « travaille à la reconnaissance du capital comme source ultime de la valeur et du marché comme son évaluation pertinente ${ }^{13}$ - porte également sur le rôle des marchés de titres négociables dans le capitalisme financiarisé. À nouveau, il s'agit de souligner la place primordiale qu'occupe désormais l'évaluation financière dans le capitalisme financiarisé sans porter de jugement sur son contenu.

10 Jean-Marie Harribey revient également sur mon article de 2015 et questionne le modèle qui y est succinctement présenté : «Le modèle d'Orléan permet-il de savoir vraiment pourquoi l'imitation "passe un seuil critique" et à quel niveau se fixe ce seuil ? ^̀̀ cette question, je réponds sans hésitation par la négative. En effet, on doit rappeler que le modèle en question n'est en rien prédictif. Il s'agit d'un « modèle conceptuel » qui a pour unique finalité de "rendre l'autonomisation de l'opinion collective conceptuellement intelligible ». Il me semble qu'en cette matière, il est réussi puisqu'on y montre qu'un groupe d'individus, tous individuellement indifférents entre deux choix $\{A\}$ et $\{B\}$, peut cependant porter son choix collectif sur l'un des deux. Cela tient à la présence d'interactions mimétiques entre les individus qui rendent possible une pluralité d'équilibres.

\section{Le modèle des prix de production}

11 Venons-en maintenant à la question centrale du prix des marchandises avant d'aborder celle, plus délicate, de la valeur qui sera ma conclusion. Sur ce point, ma réponse est très simple. Mon modèle de référence est la formulation marxienne des prix de production. Cependant, pour tenir compte de situations de concurrence imparfaite, ce modèle peut être étendu en un modèle où le prix est obtenu en ajoutant aux coûts de production une marge, fonction du pouvoir de marché de l'entreprise considérée, à la manière des post- 
keynésiens ${ }^{14}$. C'est dire qu'aux questions posées par Jean-Marie Harribey et Laurent Cordonnier sur le prix d'un avion Airbus, d'un sous-marin, d'une douzaine d'œufs, de tomates en grappe ou d'un sac à main de luxe, ma réponse ne diffère en rien de la leur. La vraie question est alors de comprendre pourquoi ceci n'a pas été compris. Deux critiques différentes m'ont été faites. La première, celle de Laurent Cordonnier, met en avant sa " frustration », légitime dès lors qu'ayant exposé ma théorie monétaire de la valeur dans L'Empire de la valeur, "je n'opère pas de retour $»^{15}$, dans la suite du livre, vers la détermination des prix. «Cette question n'intéresse-t-elle pas l'approche monétaire de la valorisation? » interroge-t-il à juste titre. Cette même critique est également faite par Matthieu Montalban qui me reproche d'avoir « délaissé la détermination des prix ». Sur ces points, je plaide coupable. Assurément, ne pas avoir explicité mon approche des prix est un manque grave, manque qui a donné lieu à d'importants malentendus car il a conduit certains à chercher à combler ce vide en me prêtant telle ou telle formulation, le plus souvent calquée sur ma théorie financière. C'est le cas de Jean-Marie Harribey qui m'impute une conception non matérialiste des prix ${ }^{16}$. Pourtant, on ne trouvera nulle part dans mes travaux de ces vingt dernières années une quelconque prise de position de ma part quant à la détermination des prix de marchandises ! Je n'en ai simplement pas parlé, comme me le reprochent à juste titre Laurent Cordonnier et Matthieu Montalban. Cette imputation sans fondement joue cependant un rôle très important dans la critique de Jean-Marie Harribey. Elle justifie sa condamnation de mon abandon de la valeur travail. L'argument est celui-ci : «en abandonnant toute théorie de la valeur fondée sur le travail », la détermination des prix perd tous ses ancrages dans la production ${ }^{17}$. On sortirait radicalement du marxisme. C'est bien le fond de la critique que Jean-Marie Harribey porte à «la vision autoréférentielle de la valeur et de la monnaie ». Ce faisant, Jean-Marie Harribey met le doigt sur un point théorique essentiel. Montrons cependant qu'il n'en est rien. Mais d'abord explicitons les raisons qui m'ont conduit à laisser de côté les prix dans L'Empire de la valeur.

12 Il me semble que la difficulté principale qu'ont rencontrée mes lecteurs a pour origine la distinction entre économie marchande et économie capitaliste, à laquelle j'accorde une importance conceptuelle cruciale. Cette distinction vient de Marx et s'illustre dans la section 1 du Capital en ce qu'elle est exclusivement consacrée à l'économie marchande. Le rapport salarial et le capitalisme ne seront introduits par Marx que dans les sections suivantes. La tradition marxiste a conservé cette distinction même si les marxistes en ont proposé des interprétations diverses ${ }^{18}$. C'est bien dans cette même tradition que je me situe. Sur ce point, mon expression dans L'Empire de la valeur est sans aucune ambiguïté comme en témoigne la citation suivante : "la réflexion de ce livre porte exclusivement sur l'économie marchande et non sur le capitalisme. S'intéresser au capitalisme supposerait d'introduire, à côté de la séparation marchande, un nouveau rapport social, à savoir le rapport salarial. Il n'en sera rien. L'analyse qui suit ignore le salariat et traite la production à la manière d'une boîte noire. $\aleph^{19} \mathrm{En}$ effet, dans la perspective que je défends, qui doit beaucoup à Suzanne de Brunhoff, la production marchande se conçoit comme étant «vide de rapports de production et de répartition entre classes sociales, elle désigne uniquement des rapports sociaux d'échange, entre producteurs de marchandises $»^{20}$. En conséquence, le concept d'économie marchande, à mes yeux, renvoie à ce qu'il y a de commun à toutes les formations sociales historiques qui connaissent la production de marchandises, quelles que soient les modalités spécifiques de cette production. C'est bien ce que nous dit Marx lorsqu'il déclare : «Peu importe que 
les marchandises soient le produit d'un système fondé sur l'esclavage, ou le produit de paysans (chinois, ryots des Indes), ou de communautés (Indes hollandaises), ou d'une production d'État (telle qu'on l'a rencontrée, fondée sur le servage aux époques anciennes de l'histoire russe), ou de peuples chasseurs demi-sauvages, etc.: c'est comme marchandise et argent qu'elles affrontent l'argent et les marchandises représentant le capital industriel. $»^{21}$ Autrement dit, quelles que soient les conditions de leur production, ce sont bien des marchandises qui ont été produites: "Quel que soit le mode de production créant les produits qui entrent dans la circulation comme marchandises, que ce soit celui de la communauté primitive ou de l'esclavage ou bien encore le mode de production petit paysan, petit bourgeois ou capitaliste, il ne change rien au caractère des produits en tant que marchandises; c'est comme marchandises que les produits doivent parcourir le procès d'échange et les modifications de forme qui l'accompagnent. $»^{22} \mathrm{Il}$ s'ensuit que Marx se refuse à identifier économie marchande et économie capitaliste contrairement à la grande majorité des économistes puisqu'à ses yeux, "circulation des marchandises et production des marchandises sont des phénomènes qui appartiennent aux modes de production les plus différents. $»^{23} \mathrm{Il}$ en découle directement qu'il n'y a aucun sens, selon moi, à chercher à produire une formule générale des prix marchands, à savoir une formule valable pour l'économie marchande en général, dès lors qu'en tant qu'économiste matérialiste, je considère que les conditions de production sont un élément essentiel du prix. Tout au contraire, je soutiens qu'il existe autant de formules de prix marchands qu'il existe de manières spécifiques de produire ces marchandises. C'est d'ailleurs ce qu'illustre avec force la formule des «prix de production» dont le domaine de validité est strictement limité à l'économie capitaliste. C'est donc précisément mon attachement à une conception matérialiste des prix qui motive ma position de ne rien dire sur les prix marchands.

Ayant fait le choix - explicite et assumé - de situer les réflexions de L'Empire de la valeur dans le cadre d'une économie marchande, et exclusivement marchande, ceci m'a conduit à laisser de côté la question de la formation du prix des marchandises parce que cette question ne lui appartient pas ${ }^{24}$. En particulier, loin de moi l'idée que les prix des marchandises seraient de nature mimétique, même si l'hypothèse mimétique peut être utile ici ou là, pour certains produits. Il se trouve cependant que la finance de marché, par le biais de la question de la liquidité, peut, pour partie, être également traitée dans ce cadre. Mais, seulement, faut-il le préciser, dans ses aspects les plus abstraits, ceux liés à la liquidité $^{25}$. La crise financière quant à elle, prise dans sa globalité, telle que l'histoire récente nous en livre de multiples exemples, reste incompréhensible si sa nature capitaliste n'est pas reconnue et se voit laissée de côté! Il m’a pourtant été reproché de défendre la position contraire sans que je ne comprenne bien pourquoi, dans la mesure où je n'ai jamais professé une telle thèse. Ceci n'est tout simplement pas ma position et ne l'a jamais été. À aucun moment, dans L'Empire de la valeur, on ne trouvera écrit que la dimension capitaliste est secondaire et que l'économiste pourrait s'en tenir aux analyses marchandes pour comprendre l'économie contemporaine. Je ne l'ai pas écrit parce que je crois le contraire, de telle sorte que, sur ce point également, je n'ai pas de désaccord avec Jean-Marie Harribey. Il m'apparaît ex post que je n'ai pas suffisamment fait connaître qu'à mes yeux, le cadre du capitalisme est bien le cadre adéquat pour penser le monde moderne. Mais simplement parce que cela me semblait aller de soi! Le lecteur doit comprendre que le fait de discuter d'un point A sans aborder le point B ne signifie nullement qu'on pense que le point $A$ est le point fondamental et que le point $B$ dont on n'a rien dit est négligeable. Par exemple, je ne parle pas d'écologie et pourtant, je pense 
que les contraintes écologiques sont fondamentales. Si je n'en dis rien, c'est parce que je considère que je n'ai pas assez de connaissances originales sur le sujet pour pouvoir en dire plus que ce que d'autres en ont déjà dit. Dans la même veine, concernant les prix des marchandises, je n'en parle pas mais ceci ne veut nullement dire que je considère la formation des prix comme secondaire. Ce n'est tout simplement pas le sujet dont je traite dans L'Empire de la valeur. Cependant, j'aurais dû expliciter ce qu'était ma position concernant la formation des prix dans le capitalisme. Enfin, dans la partie III, je m'intéresse principalement aux processus spéculatifs en tant qu'ils sont des produits de la liquidité. Je mets en avant certaines de leurs propriétés formelles (volatilité excessive, bulles, aveuglement au désastre). Le fait de les étudier dans l'abstrait ne doit pas être interprété comme impliquant que ces mécanismes seraient déconnectés des contraintes globales que connait le capitalisme.

La place me manque pour développer tous ces points. Indiquons simplement que, pour moi, une seule question est véritablement transversale à toutes les économies marchandes. Il s'agit de la question monétaire, celle-là même qui a retenu mon attention ; et non pas les prix. Ou encore, pour le dire autrement, si le concept marxiste d'économie marchande est utile théoriquement - et même indispensable - c'est en tant qu'il propose le cadre adéquat pour penser le rapport monétaire. Le fait que la monnaie s'impose dans les sociétés marchandes les plus diverses quant à leurs modalités de production apporte de l'eau à mon moulin. Cependant, même pour ce qui concerne la monnaie, soulignons que l'analyse strictement marchande atteint vite ses limites. Dès lors qu'il s'agit de rendre intelligibles des faits historiques comme l'hyperinflation allemande, le miracle du franc Poincaré, ou autres, il est nécessaire de se placer dans le cadre du capitalisme. La deuxième partie de L'Empire de la valeur, à la manière de la section 1 du Capital, s'est efforcée d'aller le plus loin possible dans l'analyse strictement marchande de la monnaie, à savoir la monnaie comme institution de la valeur économique.

On peut opposer à cette thèse le concept marxiste de valeur en ce qu'il a précisément pour objet de penser la valorisation pour toutes les économies marchandes. C'est ce que fait Jean-Marie Harribey. Avec le concept de valeur, il y aurait donc une possibilité de s'émanciper des conditions de production spécifiques des marchandises pour penser la valorisation marchande en général. Cependant, lorsqu'on s'intéresse à la manière dont les marxistes ont majoritairement interprété cette notion de valeur, il apparaît que la question est plus complexe qu'il n'y paraît. En effet, selon cette interprétation majoritaire ${ }^{26}$, Marx, dans la section 1 du Capital, aurait établi la loi de la valeur en se plaçant dans le cadre d'une "économie marchande simple", à savoir une économie d'artisans et de paysans, propriétaires de leurs moyens de production et de leurs terres, produisant pour le marché sans recourir à la main-d'œuvre salariée. À ce sujet, citons Paul Sweezy qui écrit: "Marx commence par une analyse de la "production marchande simple", autrement dit, une société dans laquelle chaque producteur est propriétaire de ses propres moyens de production et satisfait ses multiples besoins par le biais de l'échange avec d'autres producteurs se trouvant dans cette même situation ${ }^{27}$. " Comme l'écrit Marx lui-même: "L'échange de marchandises à leur valeur [...] nécessite un degré de développement moindre que l'échange aux prix de production qui requiert un développement capitaliste plus avancé ${ }^{28}$. Or, conformément à ce qu'on trouve déjà chez Adam Smith à propos de l'échange d'un castor contre un daim, en économie marchande simple, la transaction est censée se faire au prorata du temps de travail nécessaire à la production des marchandises ${ }^{29}$. C'est Engels qui remarque : «Peut-on croire que le paysan 
ou l'artisan aient été assez stupides pour échanger le produit de dix heures de travail de l'un contre celui d'une seule heure de travail de l'autre? ? ${ }^{30}$ Admettons qu'il en soit ainsi ; admettons que l'échange des marchandises se fasse conformément à leur valeur-travail lorsque s'impose la production marchande simple. Si cela est exact, en quoi est-il légitime de parler de valeur plutôt que de prix? Il me semble que ce que les marxistes qui adhèrent à l'interprétation majoritaire nomment valeur n'est rien d'autre que le prix de marché des marchandises lorsque prévaut cette forme spécifique de production qu'est la petite production marchande, de la même manière que dominent les prix de production en économie capitaliste. Je ne vois pas pour quelles raisons cette formule de prix, propre à un certain état des forces productives et des rapports de production, devrait être lue comme mettant au jour la substance même de la valeur au fondement de tous les prix. Pourquoi cette solution particulière pourrait-elle être exhaussée jusqu'au statut de principe général d'intelligibilité des prix marchands? Cette question théorique a partie liée avec ce qu'on nomme "la transformation des valeurs en prix de production", à savoir l'existence d'une formule permettant de passer des valeurs aux prix de production. En effet, la mise en évidence d'un tel lien logique serait un argument de poids pour qui cherche à établir la primauté de la valeur, en ce qu'elle autoriserait à penser le prix de production comme n'étant fondamentalement rien d'autre que de la valeur mais répartie d'une manière spécifique du fait de l'existence des classes capitaliste et salariale. C'est ce que Marx croyait avoir démontré lorsqu'il affirmait: "Toutes les variations du prix de production des marchandises se réduisent en dernière instance à un changement de valeur. $»^{31}$ En fait, le problème de la transformation s'est révélé être une impasse jusqu'à maintenant ${ }^{32}$. La reconnaissance de cette impasse doit-elle nous conduire à abandonner la loi de la valeur ? Je le crois si l'on donne à « la loi de la valeur » sa définition usuelle. Sur cette question, il est utile de présenter l'analyse que propose Arghiri Emmanuel (1972) parce qu'elle est très proche de la nôtre et, ce faisant, permet d'en bien faire comprendre la nature.

Dans L'Échange inégal, Emmanuel commence par considérer la formation du prix de marché en distinguant l'économie marchande simple et l'économie capitaliste. Il définit l'économie marchande simple précapitaliste comme une économie à un seul facteur mobile, à savoir le travail, et l'économie capitaliste, à deux facteurs mobiles, le travail et le capital. Il s'ensuit, comme il est bien connu, deux formules distinctes pour les prix de marché : la valeur-travail et le prix de production. Faut-il en déduire qu'il existerait, en conséquence, deux lois de la valeur distinctes comme Charles Bettelheim lui en fait le reproche, au nom de la théorie marxiste usuelle de la valeur ? $^{33}$ Ce n'est pas la position d'Emmanuel dès lors qu'il propose de définir la loi de la valeur d'une autre manière : « Si nous définissons la loi de la valeur comme l'allocation des ressources sociales dans les différentes branches de l'activité économique par l'intermédiaire d'une équivalence entre les produits, la réponse à cette question est manifestement négative. ${ }^{34} \mathrm{Il}$ poursuit et précise son point de vue : «Il devient alors évident que cette loi est unique et ne change point quand on passe de la valeur-travail au prix de production, puisque dans les deux cas c'est le coût individuel qui détermine l'allocation des facteurs et la division du travail dans la société. Ce qui change, ce sont les éléments de ce coût. $»^{35}$ Autrement dit, Emmanuel fait valoir que "la loi de la valeur" peut s'entendre selon deux acceptions distinctes, celle qu'il vient de proposer - loi de l'équivalence - et celle, classique, à partir du temps de travail socialement nécessaire. On trouve, nous dit-il, les deux chez Marx et Engels: "Marx et Engels emploient quelquefois le terme de "loi de la valeur" indifféremment, tantôt pour désigner en général la loi de l'équivalence (c'est-à-dire sans 
préjuger de ses déterminations), tantôt pour désigner la détermination même de cette équivalence par les quantités de travail socialement nécessaires. $»^{36}$ C'est à la première acception qu'il adhère, identifiant loi de la valeur et logique de l'équivalence. En conséquence, pour lui, l'existence de plusieurs déterminations possibles de l'équivalence ne signifie pas qu'il y ait plusieurs lois de la valeur. Son appréciation négative de la seconde acception - la valeur comme la quantité de travail socialement nécessaire contenue dans une marchandise - procède d'une critique dont l'esprit est très proche des analyses que j'ai développées dans L'Empire de la valeur ${ }^{37}$. En effet, il écrit à propos de cette unicité de la loi de la valeur : "Cette unicité est celle de la "valeur" (en tant que quantité de travail socialement nécessaire), conçue comme une substance $e^{38}$, pour ainsi dire injectée dans la marchandise pendant la production et l'accompagnant ensuite comme sa propriété inhérente, de sorte que la valeur relative, quelle qu'elle soit, ne serait qu'une forme phénoménale, visible, apparaissant au niveau de la circulation, de cette valeur absolue, objective, invisible, créée au cours de la production. $»^{39}$ Par conséquent, « le prix de production ne serait selon cette conception qu'un des avatars de cette essence immuable, une sorte de distribution différente du même jeu de cartes. $»^{40}$ Emmanuel situe explicitement son désaccord avec Bettelheim sur la question de ce qu'il nomme désormais la «valeur absolue », c'est-à-dire sur l'idée d'une valeur offrant l'intelligibilité de toutes les formes de prix de marché, quels que soient les rapports de production! Cette position critique à l'égard de la valeur est très exactement celle que je défends dans L'Empire de la valeur lorsque je critique ce que je nomme "l'hypothèse substantialiste ». Il n'y a pas de valeur absolue mais un ensemble de prix dont les formules varient en fonction des conditions de production. C'est cela que je nomme « conception matérialiste des prix ».

Il découle de ces réflexions l'affirmation suivante qui est une réponse directe à la critique de Jean-Marie Harribey : le fait d'abandonner «toute théorie de la valeur fondée sur le travail $»^{41}$ ne me conduit nullement à renoncer à l'approche matérialiste des prix. Mieux encore, comme toute l'analyse du capitalisme menée par Marx dans Le Capital repose sur l'hypothèse des prix de production, à laquelle je souscris, rares sont ses conclusions ou ses résultats que mon cadre d'analyse serait amené à rejeter pour des raisons de nature conceptuelle. C'est principalement autour de la question monétaire que les divergences pourraient être les plus notables, mais il se trouve que cette question ne joue pas un grand rôle dans Le Capital, si l'on met de côté sa première section qui lui est spécifiquement consacrée. J'écris cela parce que quelquefois, à lire Jean-Marie Harribey, il pourrait sembler que mes analyses du capitalisme sont à des années-lumière des analyses classiques du marxisme, alors même que, sur une majorité de points, je les partage.

\section{L'approche monétaire de la valeur}

Enfin, venons-en à la valeur. Sur ce point également, l'approche d'Emmanuel ne manque pas d'intérêt, qui fait de l'équivalence la loi fondamentale des économies marchandes. J'adhère à cette idée : "Partout où les rapports marchands se produisent, il s'établit à travers le marché une équivalence, c'est-à-dire une réduction de la qualité à la quantité, autrement dit, une quantification de valeurs d'usage qualitativement différentes. Cette équivalence s'exprime au niveau du phénomène par le prix de marché. $»^{42}$ Mais peut-on penser cette commensurabilité - pour reprendre le terme qu'utilise Marx - autrement que comme l'expression d'une substance commune, partagée par toutes les marchandises, ce qu'on nomme la valeur? Emmanuel s'y refuse. La seule réalité pour lui, 
ce sont les prix de marché. Mais, ce faisant, il laisse la question de l'équivalence marchande non traitée. Pourtant, il y a bien quelque chose de commun à toutes les économies marchandes, au-delà des prix spécifiques qui s'y forment lorsque prévaut tel ou tel rapport de production, à savoir la loi de l'équivalence elle-même. Ne mérite-t-elle pas une analyse ? D'où vient l'institution de l'équivalence? La réponse que je propose est la suivante: l'équivalence marchande résulte du désir que tous les producteurs échangistes éprouvent à l'égard de la monnaie, désir qu'on trouve dans toutes les économies marchandes, quelles que soient par ailleurs les conditions de production de ces marchandises, comme en atteste l'existence universelle de la monnaie marchande au sein $\mathrm{de}^{43}$. Dans ce cadre, la valeur d'une marchandise se confond avec la quantité de monnaie que le producteur peut obtenir lorsqu'il la vend. Autrement dit, la monnaie institue un espace abstrait de mesure, l'espace des prix. Ma thèse est qu'il est erroné de vouloir chercher à penser cette mesure comme l'expression d'une autre grandeur dont elle pourrait être dérivée, comme l'utilité, la rareté ou le travail. Cet espace est sui generis ; il est une production de la monnaie elle-même. C'est un nouvel ordre de valeurs qui se trouve institué, irréductible aux autres valeurs, en conflit et/ou en complémentarité avec elles. Ce faisant, se donne à voir une commensurabilité qui échappe à l'hypothèse substantialiste. Elle peut être dite autoréférentielle par le fait qu'elle ne renvoie qu'à ellemême, comme l'est également l'espace du droit ou de la religion ${ }^{44}$.

Pour autant, cela ne signifie en aucun cas que les prix qui s'y forment soient sans rapport avec les rapports de production! C'est ce que montrent sans ambiguïté les formules de prix propres à la production marchande simple ou propres au capitalisme qui ont été exhibées ${ }^{45}$; ce qui suffit à contredire la critique que porte Jean-Marie Harribey à l'encontre de mon approche. Il n'y a pas lieu de s'en étonner. L'espace abstrait des prix définit un espace de valeur sur lequel les conflits entre individus et entre classes vont trouver naturellement à s'exprimer. Il s'agit, en effet, pour chacun de faire valoir ses droits au partage du produit économique de la société, droits exprimés monétairement. Ainsi, par exemple, dans le cadre du capitalisme, le capital cherchera-t-il à accroître ses profits et les salariés, leur salaire. De même, dans le cadre de la production marchande, comme l'écrit Emmanuel : «Si l'homme s'est mis un jour à comparer des choses aussi dissemblables qu'une pirogue et une vache, ce fut uniquement pour pouvoir rémunérer au plus juste le travail des producteurs de l'une et de l'autre. $»^{46}$ Pour cette raison, on comprend sans difficulté que les prix, loin d'être des constructions fantasmées, soient tout au contraire très sensibles aux conditions de la production comme aux conflits sociaux. Autrement dit, ce n'est pas parce que, dans mon approche, la valeur ne s'identifie pas au travail que les conflits qu'elle suscite sont moins réels. De même, malgré leur caractère très abstrait, les conflits religieux, par exemple quant à la nature du père, du fils et du Saint-Esprit, qui ont fait rage au début du christianisme, recouvraient des enjeux de pouvoir tout ce qu'il y a de plus réel. L'intensité des conflits n'est pas fonction de la supposée substantialité de la valeur qui est disputée mais de la place de cette valeur dans la hiérarchie des valeurs de la société considérée.

\section{Conclusion}

20 Je terminerai en remerciant Jean-Marie Harribey de l'intérêt qu'il a toujours manifesté pour mes travaux. Ses critiques portent sur des points théoriques très importants pour la tradition marxiste. Il était normal que mes propositions, qui rompent en grande partie 
avec cette tradition pour ce qui est de la théorie de la valeur et de la monnaie, suscitent ainsi des réserves voire des rejets. Mais j'espère avoir montré que mon projet ne se voulait nullement une liquidation du marxisme. Bien au contraire, dans différents textes ${ }^{47}$ , je me suis efforcé de montrer que l'approche que je proposais ne dérogeait pas aux principes fondamentaux de l'analyse marxiste. Je propose même d'y voir un approfondissement dont l'axe serait de penser la monnaie comme l'objet d'un conflit entre les producteurs, là où Marx y voit un contrat entre marchandises. Mais c'est là un autre débat ${ }^{48}$.

\section{BIBLIOGRAPHIE}

Brunhoff Suzanne (de) (1974), La Politique monétaire. Un essai d'interprétation marxiste, Paris, Puf, coll. SUP « L'économiste », $\mathrm{n}^{\circ} 30$.

Cordonnier Laurent (2012), " Une théorie de la valorisation », Notes critiques consacrées à L'Empire de la valeur, Revue Française de Socio-Économie, n 10, 2012/2, p. 289-294.

DiQuattro Arthur (2007), " The Labor Theory of Value and Simple Commodity Production », Science \& Society, vol. 71, $\mathrm{n}^{\circ}$ 4, octobre, p. 455-483.

Durkheim Émile (1993) [1895], Les Règles de la méthode sociologique, Puf, coll. « Quadrige ».

Emmanuel Arghiri (1972), L'Échange inégal. Essai sur les antagonismes dans les rapports économiques internationaux, Paris, François Maspéro, complété de « Préface et remarques théoriques de Charles Bettelheim », suivi de la réponse d'Arghiri Emmanuel.

Engels Friedrich (1974) [1895], « Complément et supplément au livre III du Capital » in Karl Marx, Le Capital, Livre troisième, Tome I, Paris, Éditions Sociales, p. 26-44.

Harribey Jean-Marie, « André Orléan, L’Empire de la valeur. Refonder l'économie, Paris, Seuil, 2011 », Revue de la régulation, [En ligne], 10 | 2e semestre / Autumn 2011, mis en ligne le 21 décembre 2011, consulté le 30 mars 2018. URL : http://journals.openedition.org/regulation/9483 Harribey Jean-Marie (2013), La Richesse, la valeur et l'inestimable. Fondements d'une critique socioécologique de l'économie capitaliste, Paris, Les Liens qui Libèrent.

Lavoie Marc (2006), Introduction to Post-Keynesian Economics, Palgrave Macmillan.

Marx Karl (1971), Le Capital, Livre Premier, Tome I, Paris, Éditions sociales (traduction de Joseph Roy entièrement révisée par l'auteur). Manquent dates originales

Marx Karl (1950), Le Capital, Livre Premier, Tome III, Paris, Éditions sociales (traduction de Joseph Roy entièrement révisée par l'auteur).

Marx Karl (1960), Le Capital, Livre Deuxième, Tome I, Paris, Éditions sociales.

Montalban Matthieu (2012), « De la place de la théorie de la valeur et de la monnaie dans la théorie de la régulation : critique et synthèse ", Revue de la Régulation, $\mathrm{n}^{\circ} 12,2^{\mathrm{e}}$ semestre [http:// journals.openedition.org/regulation/9797]

Orléan André (1999), Le Pouvoir de la finance, Paris, Odile Jacob. 
Orléan André (2011a), L'Empire de la valeur. Refonder l'économie, Paris, Le Seuil, coll. « La couleur des idées ".

Orléan André (2011), « Réponse à Jean-Marie Harribey », Revue de la régulation, [En ligne], $\mathrm{n}$ - 10 | 2e semestre / Autumn 2011, mis en ligne le 21 décembre 2011, consulté le 30 mars 2018. URL : http://journals.openedition.org/regulation/9502

Orléan André (2013), « Entretien avec André Orléan », Revue de la Régulation, [En ligne], nº 14 | 2e semestre / Autumn 2013, mis en ligne le 12 décembre 2013, consulté le 30 mars 2018. URL : http://journals.openedition.org/regulation/10305 ; DOI : 10.4000/regulation.10305

Orléan André (2015), « La valeur économique comme fait social : la preuve par les évaluations boursières ", Revue de la régulation, [En ligne], $\mathrm{n}^{\circ} 18$ | 2e semestre / Autumn 2015, mis en ligne le 20 décembre 2015, consulté le 30 mars 2018. URL : http://journals.openedition.org/ regulation/11441; DOI : 10.4000/regulation.11441

Orléan André (2017), « Réflexions sur la théorie marxiste de la monnaie », in Catherine ColliotThélène (éd.), Que reste-t-il de Marx ?, Rennes, Presses universitaires de Rennes, p. 93-116.

Orléan André (2018), « De quelques débats à propos de la production marchande chez Marx », à paraître dans l'ouvrage collectif : Monnaie Finance et Capital. Contributions en l'honneur de Suzanne de Brunhoff aux Presses universitaires de Rennes, sous la direction de Cédric Durand.

Samuelson Paul A. (1970), « The "Transformation" from Marxian "Values" to Competitive "Prices": A Process of Rejection and Replacement ", Proceeding of the National Academy of Sciences, vol. $67, \mathrm{n}^{\circ} 1,15$ September, p. 423-425.

Sweezy Paul M. 1962 [1942], The Theory of Capitalist Development. Principles of Marxian Political Economy, London, Dennis Dobson.

\section{NOTES}

1. L'autre représentant de ce que Jean-Marie Harribey nomme « la vision autoréférentielle de la valeur et de la monnaie » est Frédéric Lordon, à la critique duquel est consacrée une large partie de la seconde section de l'examen critique de Jean-Marie Harribey, intitulée «La monnaie, rapport social ».

2. Dans plusieurs textes, Jean-Marie Harribey a déjà fait connaître sa critique de ma conception de la valeur : Harribey (2011) et Harribey (2013). J’y ai répondu une première fois dans Orléan (2011a).

3. Se reporter à L'Empire de la valeur.

4. J'écris «communément interprétée par la tradition marxiste" car nous verrons Arghiri Emmanuel (1972) proposer une autre conception de la loi de la valeur marxiste. Cette question fait l'objet d'un débat passionnant et instructif entre Arghiri Emmanuel et Charles Bettelheim. Se reporter à Emmanuel (1972).

5. Cordonnier (2011, p. 294).

6. Ce point est largement développé au chapitre VI de L'Empire de la valeur.

7. p. 176-177.

8. Le Pouvoir de la finance, p. 183.

9. Le « retrouve " dans « on retrouve ici » ne renvoie pas à l'analyse générale mais aux exemples précédents.

10. Le « On retrouve » au début de la citation renvoie, non pas à la finance en général, mais aux premiers exemples de panique financière présentés dans cette même section.

11. Ibid., p. 182. 
12. Le Pouvoir de la finance, p. 245-246.

13. Ibid., p. 249.

14. Se reporter à Lavoie (2006).

15. Cordonnier, p. 294.

16. Notons que ce n'est pas le cas de Laurent Cordonnier et Matthieu Montalban. Leur critique s'arrête au fait que rien n'est dit sur la détermination des prix.

17. Il faut ici noter, sans avoir la place de le développer, que, chez Marx, le travail créateur de valeur, c'est le travail abstrait. On sait quelle importance Marx accorde à la distinction entre travail concret et travail abstrait. En conséquence, la théorie de la valeur-travail marxiste ne peut être considérée comme établissant un lien entre prix et conditions de production, comme le défend Jean-Marie Harribey, qu'à la condition qu'on sache passer du travail concret au travail abstrait - ce qui n'est pas le cas car « c'est l'échange seul qui opère cette réduction » (Marx, Livre premier, tome I, 1971, p. 86)!

18. Se reporter à Orléan (2018). Texte transmissible sur simple demande.

19. Orléan (2011a), p. 23.

20. Brunhoff (1974), p.55. Elle dit également: «Prise dans son sens littéral, la production marchande $[. .$.$] n'est que production de marchandises » (p. 52).$

21. Le Capital, Livre deuxième, tome 1, p. 101-102.

22. Le Capital, Livre premier, tome III, chapitre XX, p. 334.

23. Le Capital, Livre premier, Tome I, p. 122, note 1 (Éditions sociales).

24. Par-delà ses aspects les plus abstraits, bien évidemment.

25. Il est vrai cependant qu'on est ici à la limite de notre hypothèse car, à l'évidence, lorsqu'on parle de finance, on parle de profits et donc de capitalisme. Aussi, dans la partie III de L'Empire qui est consacrée à la finance de marché, c'est bien du capitalisme dont il est question, même si mon analyse a pour objet spécifique la liquidité.

26. Sur cette interprétation majoritaire et ses critiques, se reporter à Orléan (2018).

27. Sweezy (1962), p. 23.

28. Cité par Engels (1974), p. 31.

29. Notons que cette question est en grande partie une question empirique. Il s'agit d'observer comment les prix se forment en économie marchande simple. DiQuattro (2007) s'y est attelé. Il a cherché à vérifier si la valeur travail permettait effectivement de rendre compte de la formation des prix en économie marchande simple. Il conclut de ses observations qu'il n'en est rien. Sur ce point également, on peut lire Orléan (2018).

30. Engels (1974), p. 33.

31. Capital, Livre 3, Tome I, p. 220. Sur l'erreur de Marx, se reporter à Emmanuel (1972).

32. Ce sujet peut remplir une bibliothèque. C'est dire que la place manque pour expliciter cette position. Rappelons la fameuse citation de Samuelson (1970) : «En résumé la "transformation" des valeurs en prix peut être décrite logiquement par le biais de la procédure suivante : (1) Écrire les relations en valeurs ; (2) prendre une gomme et les effacer ; (3) écrire les relations en prix - c'est ainsi que procède la soi-disant transformation » (p. 425).

33. Ibid., « Dans le mode de production capitaliste, la loi de la valeur agit comme loi de formation des prix de production si bien qu'il n'est pas possible d'opposer valeur et prix de production. Le prix de production est l'effet de la loi de la valeur opérant dans le mode de production capitaliste » p. 302.

34. Ibid., p. 346.

35. Ibid., p. 347 et 348 .

36. Ibid., note 1 , p. 343.

37. Je n'avais pas lu Emmanuel (1972) à cette époque.

38. Qui est bien une substance sociale, comme le souligne Jean-Marie Harribey.

39. Ibid., p. 352. 
40. Ibid., p. 352.

41. Harribey...

42. p. 343.

43. Bien évidemment, je ne peux ici développer la nature du désir de monnaie. On peut se reporter à la partie II de L'Empire de la valeur. J'indique ici simplement que ce désir trouve son origine dans la nature des rapports marchands et non pas dans la psychologie des acteurs. À ce sujet, comment ne pas citer Durkheim : « Toutes les fois qu'un phénomène social est directement expliqué par un phénomène psychique, on peut être assuré que l'explication est fausse.»

44. Se reporter à la notion d'autopoièse qu'on doit à Humberto R. Maturana et Francisco J. Varela. Reprise par Niklas Luhmann.

45. Il est bien clair que cette question des prix est, en large partie, une question empirique. Nous en sommes tout à fait conscients mais, dans le cadre de cette contribution qui répondait à des critiques d'ordre théorique, nous sommes restés exclusivement dans ce registre théorique. Soulignons cependant que DiQuattro (2007) a cherché à vérifier si la valeur travail permettait effectivement de rendre compte de la formation des prix en économie marchande simple. Il conclut de ses observations qu'il n'en est rien. Sur ce point également, on peut lire Orléan (2018).

46. Emmanuel (1970), p. 58.

47. Orléan (2013 et 2017).

48. C'est tout l'objet de : Orléan (2017).

\section{AUTEUR}

\section{ANDRÉ ORLÉAN}

Directeur de recherche au CNRS, UMR Paris-Jourdan sciences économiques, orlean@pse.ens.fr 